Draft version MARCh 20, 2018

Preprint typeset using $\mathrm{L}^{\mathrm{A}} \mathrm{T}_{\mathrm{E}} \mathrm{X}$ style emulateapj v. 01/23/15

\title{
PRANDTL-NUMBER EFFECTS IN HIGH-RAYLEIGH-NUMBER SPHERICAL CONVECTION
}

\author{
Ryan J. Orvedahl ${ }^{1}$, Michael A. Calkins ${ }^{2}$, Nicholas A. Featherstone ${ }^{3,5}$, Bradley W. Hindman ${ }^{1,4}$ \\ ${ }^{1}$ Department of Astrophysical \& Planetary Sciences, University of Colorado, Boulder, CO 80309-0391, USA \\ ${ }^{2}$ Department of Physics, University of Colorado, Boulder, CO 80309-0390, USA \\ ${ }^{3}$ Department of Applied Mathematics, University of Colorado, Boulder, CO 80309-0526, USA \\ ${ }^{4}$ JILA, University of Colorado, Boulder, CO 80309-0440, USA and \\ ${ }^{5}$ Research Computing, University of Colorado, Boulder, CO 80309-0440, USA \\ Draft version March 20, 2018
}

\begin{abstract}
Convection is the predominant mechanism by which energy and angular momentum are transported in the outer portion of the Sun. The resulting overturning motions are also the primary energy source for the solar magnetic field. An accurate solar dynamo model therefore requires a complete description of the convective motions, but these motions remain poorly understood. Studying stellar convection numerically remains challenging; it occurs within a parameter regime that is extreme by computational standards. The fluid properties of the convection zone are characterized in part by the Prandtl number $\operatorname{Pr}=\nu / \kappa$, where $\nu$ is the kinematic viscosity and $\kappa$ is the thermal diffusion; in stars, $\operatorname{Pr}$ is extremely low, $\operatorname{Pr} \approx 10^{-7}$. The influence of $\operatorname{Pr}$ on the convective motions at the heart of the dynamo is not well understood since most numerical studies are limited to using $\operatorname{Pr} \approx 1$. We systematically vary $\operatorname{Pr}$ and the degree of thermal forcing, characterized through a Rayleigh number, to explore its influence on the convective dynamics. For sufficiently large thermal driving, the simulations reach a so-called convective free-fall state where diffusion no longer plays an important role in the interior dynamics. Simulations with a lower Pr generate faster convective flows and broader ranges of scales for equivalent levels of thermal forcing. Characteristics of the spectral distribution of the velocity remain largely insensitive to changes in Pr. Importantly, we find that Pr plays a key role in determining when the free-fall regime is reached by controlling the thickness of the thermal boundary layer.

Subject headings: Sun: helioseismology - Sun: interior - Sun: magnetic fields -stars: fundamental parameters - stars: interiors - stars: kinematics and dynamics
\end{abstract}

\section{INTRODUCTION}

Convective motions within the outer one third of the Sun transport energy from the radiative interior to the photosphere. In the process, these overturning motions, which are thought to drive the solar differential rotation, play a pivotal role in generating solar magnetic field. Any model of the solar dynamo necessarily requires a description of the Sun's underlying convective motions, and yet those motions remain poorly characterized in spite of the observational coverage enabled by the Sun's proximity to Earth.

On the largest spatial scales, photospheric convection manifests in celluar patterns known as supergranules that were first noted by Hart (1954) and better characterized by Leighton et al. (1962). The horizontal extent of these cells is approximately $35 \mathrm{Mm}$, and they possess a spectral peak in photospheric Dopplergram power around spherical harmonic degree $\ell \approx 120$ (e.g., Hathaway et al. 2000 . 2015). In addition, smaller-scale motions known as granulation are clearly visible in the photosphere, possessing a characteristic size of about $1 \mathrm{Mm}$ and a clear peak in photospheric Dopplergram power around $\ell \approx 10^{3}$ (Bray et al. (1984).

Presently, only the granular component of photospheric convection is reliably captured in direct numerical simulations. Radiative hydrodynamic simulations of solar surface convection that can simulate granulation fail to yield clear evidence for supergranulation (e.g., Stein et al. 2009, Ustyugov 2010). Inconsistencies between numerical models and the Sun have also been ob- served in the velocity power distribution associated with larger scales of convection (e.g., Miesch et al.|2008). Measurements of deep convective flow speeds, made using time-distance helioseismology, suggest that convection models may overestimate the amplitude of the convection on spatial scales larger than $30 \mathrm{Mm}$ (Hanasoge et al. 2012). The results of Hanasoge et al. (2012) estimate that the convective velocities on spatial scales larger than $70 \mathrm{Mm}$ is at most $5-6 \mathrm{~m} \mathrm{~s}^{-1}$, about an order of magnitude weaker than that expected from simulations or theoretical arguments (e.g., Miesch et al. 2012). Ring-analysis measurements of the subsurface flows in the near-surface shear layer, however, exhibit good agreement with models and theory (Greer et al. 2015).

Resolving these descrepencies requires careful comparison of these two different helioseismic techniques, and perhaps improvements to both. A resolution to this problem also requires a better theoretical understanding of convective dynamics under stellar conditions. Exploring stellar convection numerically remains challenging owing to the fact that it occurs within parameter regimes that are considered extreme by modern computational and laboratory standards alike. These regimes can be characterized by several nondimensional parameters. In particular, the Reynolds number measures the relative importance of inertial forces to viscous forces, the Rayleigh number expresses the relative strength of buoyancy driving and diffusive effects, and the Prandtl number specifies the relative importance of viscosity to thermal diffusion. Estimates for the Sun lead to values 
of the Reynolds, Rayleigh, and Prandtl numbers on the order of $10^{13}, 10^{20}$, and $10^{-7}$, respectively, indicating the solar convection zone is highly turbulent (e.g., Ossendrijver 2003).

Such extreme values of nondimensional parameters are largely an expression of the fact that while diffusion in stellar interiors may be active on very small scales, it tends to be negligible at the system scale. Achieving such a situation, wherein diffusion plays no appreciable role in the leading-order force balance, is possible in those parameter regimes already accessible through computational models. Featherstone \& Hindman (2016b a) identified two such regimes by exploring the response of convection to changes in Rayleigh number and Ekman number (which expresses the relative importance of the Coriolis force and viscous diffusion), while using a fixed Prandtl number of unity. In so doing, they identified asymptotic scaling laws for spectral properties of the convection that could in principle be extrapolated to the stellar parameter regime. The purpose of this paper is to extend those studies by exploring the response of the convective spectrum to changes in the Prandtl number.

This paper is organized as follows. In Section 1.1 . we summarize earlier investigations into the role of the Prandtl number. In Sections 2 and 3 , we discuss our numerical model and the parameter space that was explored. Results are discussed in Section 4 followed by a discusion of their implications in Section 5.

\subsection{Prandtl Number in Convection}

The Prandtl number $\operatorname{Pr}=\nu / \kappa$, where $\nu$ is the kinematic viscosity and $\kappa$ is the thermal diffusivity, is known to be small in the dynamo regions of planets and stars (Ossendrijver 2003, Roberts 2007). It is well known from linear theory that the value of the Prandtl number both influences the structure and amplitude of convective motions and controls the critical Rayleigh number required for the onset of rotating convection (e.g., Chandrasekhar 1961). Asymptotic approximations to the governing fluid equations can be carried out based on the size of the Prandtl number. Such approximations yield some insight into the convective dynamics arising under different Prandtl number regimes. In the large Prandtl number limit, the influence of inertia is weak and can be neglected; this limit is routinely exploited in studying the convection of planetary mantles where the Prandtl number can be of order $10^{20}$ (Schubert et al. 2001). Spiegel (1962) developed an approximate set of equations valid in the limit $\mathrm{Pr} \rightarrow 0$ that showed inertia plays a leadingorder role in the convective dynamics (see also Thual 1992). Both of these approximate models have been applied only to nonrotating and incompressible Boussinesq systems to date.

Much less is known about the role of the Prandtl number in compressible convection. Earlier studies of compressible convection have primarily used Prandtl number of order unity (e.g., Gilman 1977; Gilman \& Glatzmaier 1981; Goudard \& Dormy 2008; Christensen 2011; Soderlund et al. 2012: Schrinner et al. 2012, Gastine et al.|2015, 2016 (Wicht \& Meduri 2016). While there are also many studies that make use of nonunity Prandtl numbers (e.g. Brown et al. 2011; Käpylä et al. 2013; Nelson et al. 2014; Jones |2014; Augustson et al. |2015, 2016; Duarte et al. 2016 Brun et al. 2017), no parameter studies that vary the Prandtl number systematically have been carried out. One exception to this trend is the work of O'Mara et al. (2016), who explored the characteristics of high Prandtl compressible convection. These authors found that high Prandtl convection tended to possess lower characteristic flow-speeds with respect to unity Prandtl number convection, owing to the enhanced entropy content of its downflow plumes. Finally, we note that recent work using a small Prandtl number with rapid rotation has found that the anelastic approximation can yield spurious behavior (Calkins et al. 2015a) that does not appear in nonrotating anelastic convection (Calkins et al. 2015b). These results raise serious questions regarding the applicability of the anelastic approximation within rotating stellar interiors where it remains to be seen if the convective flows are well-approximated by a Prandtl number of unity.

Systematic parameter space studies of convective dynamics in stellar interiors have, so far, focused largely on the role of buoyancy driving and rotation. Featherstone \& Hindman (2016b) investigated the response of the convection to varying Rayleigh number and varying degrees of density stratification. Those simulations were nonrotating, hydrodynamic, $\mathrm{Pr}=1$ and demonstrated a clear scaling relationship between kinetic energy and Rayleigh number. Those results also suggest that a naive interpretation of model results (by ascribing solar values to all problem parameters but the diffusion coefficients) will naturally overestimate the low-wavenumber power in the convective power spectrum. The influence of rotation was investigated using a similar methology by Featherstone \& Hindman (2016a) who identified a complementary scaling law relating convective-cell-size and rotational influence. When rotation is present, and diffusive effects are negligible, the typical spatial-size of convective cells is determined primarily through the Rossby number, which expresses the ratio of the rotation period to a characteristic convective time scale. Their work was also restricted to $\mathrm{Pr}=1$.

Through this paper, we extend these studies and examine the effects of Prandtl number variation on the convective dynamics. We present a series of numerical simulations designed to examine how the structure and amplitude of the convective flow within a stellar interior depends on the Prandtl number and the convective forcing. We vary the Prandtl number and the convective forcing in a systematic way, covering both low and high Prandtl numbers. Effects due to rotation and magnetism are not included. We will show that the convection develops smaller-scale structures as the convective forcing is increased and the Prandtl number is decreased, corresponding to an increase in highwavenumber power. As the high-wavenumber power increases, the low-wavenumber power decreases and this trend occurs for all Prandtl numbers studied. We also show that the Prandtl number has an important influence on the boundary-layer thickness.

\section{NUMERICAL MODEL}

This study is based on a series of $3 \mathrm{D}$, nonlinear convection simulations that use the pseudo-spectral convection code Rayleigh (e.g., Featherstone \& Hindman 2016b). We employ a spherical geometry and represent the horizontal variation of all variables along spherical surfaces using spherical harmonics $Y_{\ell}^{m}(\theta, \phi)$. Here $\ell$ is the spherical 
harmonic degree, and $m$ is the azimuthal mode order. In the radial direction, we employ a Chebyshev collocation method, expanding all variables in Chebyshev polynomials $T_{n}(r)$, where $n$ is the degree of the polynomial.

We are particularly interested in understanding convection in the deep stellar interior, far removed from the photosphere. In this region, plasma motions are subsonic and perturbations to thermodynamic variables are small compared to their mean, horizontally averaged values (represented using overbars). Under these conditions, the anelastic approximation provides a convenient description of the system's thermodynamics (Gough 1969 Gilman \& Glatzmaier 1981). The governing evolution equations include the continuity equation

$$
\boldsymbol{\nabla} \cdot(\bar{\rho} \boldsymbol{u})=0,
$$

where $\bar{\rho}$ is the background density and $\boldsymbol{u}$ is the fluid velocity. The momentum equation is given by

$$
\frac{D \boldsymbol{u}}{D t}=-\boldsymbol{\nabla} \frac{P}{\bar{\rho}}-\frac{S}{c_{p}} \boldsymbol{g}+\frac{1}{\bar{\rho}} \boldsymbol{\nabla} \cdot \mathcal{D},
$$

where $P$ is the pressure, $S$ is the entropy, $c_{p}$ is specific heat at constant pressure, $\boldsymbol{g}$ is the gravitational acceleration, and the viscous stress tensor $\mathcal{D}$ is given by

$$
\mathcal{D}_{i j}=2 \bar{\rho} \nu\left[e_{i j}-\frac{1}{3} \partial_{k} u_{k} \delta_{i j}\right] .
$$

Here, $e_{i j}$ is the strain rate tensor and $\delta_{i j}$ is the Kronecker delta. Written in terms of the entropy, the thermal energy equation is given by

$$
\begin{aligned}
\bar{\rho} \bar{T} \frac{D S}{D t}= & \nabla \cdot(\bar{\rho} \bar{T} \kappa \nabla S)+Q(r, \theta, \phi) \\
& +2 \bar{\rho} \nu\left[e_{i j} e_{i j}-\frac{1}{3}(\boldsymbol{\nabla} \cdot \boldsymbol{u})^{2}\right],
\end{aligned}
$$

where $\bar{T}$ is the background temperature. Sources of internal heating and cooling are encapsulated in the functional form of $Q$. A linearized equation of state closes the system and is given by

$$
\frac{\rho}{\bar{\rho}}=\frac{P}{\bar{P}}-\frac{T}{\bar{T}}=\frac{P}{\gamma \bar{P}}-\frac{S}{c_{p}},
$$

assuming the ideal gas law

$$
\bar{P}=\mathcal{R} \bar{\rho} \bar{T},
$$

where $\mathcal{R}$ is the gas constant and $\gamma=5 / 3$ is the adiabatic index.

\section{NUMERICAL EXPERIMENT}

We have constructed a set of 34 model stellar convection zones designed to explore how the convective kinetic energy depends on both the thermal diffusion and the viscous diffusion as characterized by the Prandtl number. The diffusion coefficients are taken to be constant values within each simulation. In particular, they have no variation with radius. Table 1 has a detailed list of all model parameters for each run.

Each model is constructed using a polytropic background state following Jones et al. (2011). The background states were constructed in a similar fashion to the models presented in Featherstone \& Hindman (2016b).
We use a polytropic index of $n=1.5$, which corresponds to the adiabatic value, and model the innermost 3 density scale heights of the convection zone. The spherical shell has an aspect ratio of $\chi=r_{i} / r_{o}=0.759$ corresponding to a dimensional shell depth of $159 \mathrm{Mm}$, where $r_{i}$ and $r_{o}$ are the inner and outer radii of the domain, respectively.

Our models are fully characterized by two parameters: a Rayleigh number and a Prandtl number. As discussed in Featherstone \& Hindman (2016b), a flux Rayleigh number $\mathrm{Ra}_{F}$ appropriate for this system may be defined as

$$
\operatorname{Ra}_{F}=\frac{\tilde{g} \tilde{F} H^{4}}{c_{p} \tilde{\rho} \tilde{T} \nu \kappa^{2}},
$$

where tildes indicate volume averages over the full shell, making $\mathrm{Ra}_{F}$ a bulk Rayleigh number. In this definition, $F$ is the thermal energy imposed by the radiative heating and $H$ is chosen to be the shell depth, $r_{o}-r_{i}$. The nondimensionalization was carried out using the shell depth and the viscous diffusion timescale $H^{2} / \nu$.

Heat enters the system through the internal deposition by $Q$, which drops to zero at the upper boundary. In all simulations we adopt a functional form of $Q$ that depends only on the background pressure profile such that

$$
Q(r, \theta, \phi)=\alpha\left(\bar{P}(r)-\bar{P}\left(r_{o}\right)\right) .
$$

The normalization is defined so that

$$
L_{\star}=4 \pi \int_{r_{i}}^{r_{o}} Q(r) r^{2} \mathrm{~d} r,
$$

where $L_{\star}$ is the stellar luminosity. The thermal energy flux $F(r)$ that convection and conduction must transport across a spherical surface at radius $r$ is then given by

$$
F(r)=\frac{1}{r^{2}} \int_{r_{i}}^{r} Q(x) x^{2} \mathrm{~d} x .
$$

For all simulations, we have adopted impenetrable and stress-free boundary conditions on the velocity. The radial entropy gradient is forced to vanish at the lower boundary of the convection zone, and the entropy perturbations are forced to vanish at the upper boundary.

Our numerical experiments span the range of $4 \times 10^{2} \lesssim$ $\mathrm{Ra}_{F} \lesssim 7 \times 10^{6}$ and $0.1 \leq \operatorname{Pr} \leq 4$. Each simulation was initialized using a small random thermal perturbation, evolved until the kinetic energy reached a statistically steady state, and further evolved for at least one diffusion time. Since there are two diffusion time scales, the larger of the two was used for this purpose. The larger of the two diffusion times is also the time interval over which a time average is computed when necessary. This averaging interval includes several tens of convective overturning times.

\section{SURVEY OF RESULTS}

\subsection{Kinetic Energy Scaling}

We begin our examination of the convective energetics by looking at the integrated kinetic energy KE, defined as

$$
\mathrm{KE}=\frac{1}{2} \int \bar{\rho}(r)|\boldsymbol{u}(r, \theta, \phi)|^{2} \mathrm{~d}^{3} x,
$$

where the integration is computed over the entire domain and then time averaged. 

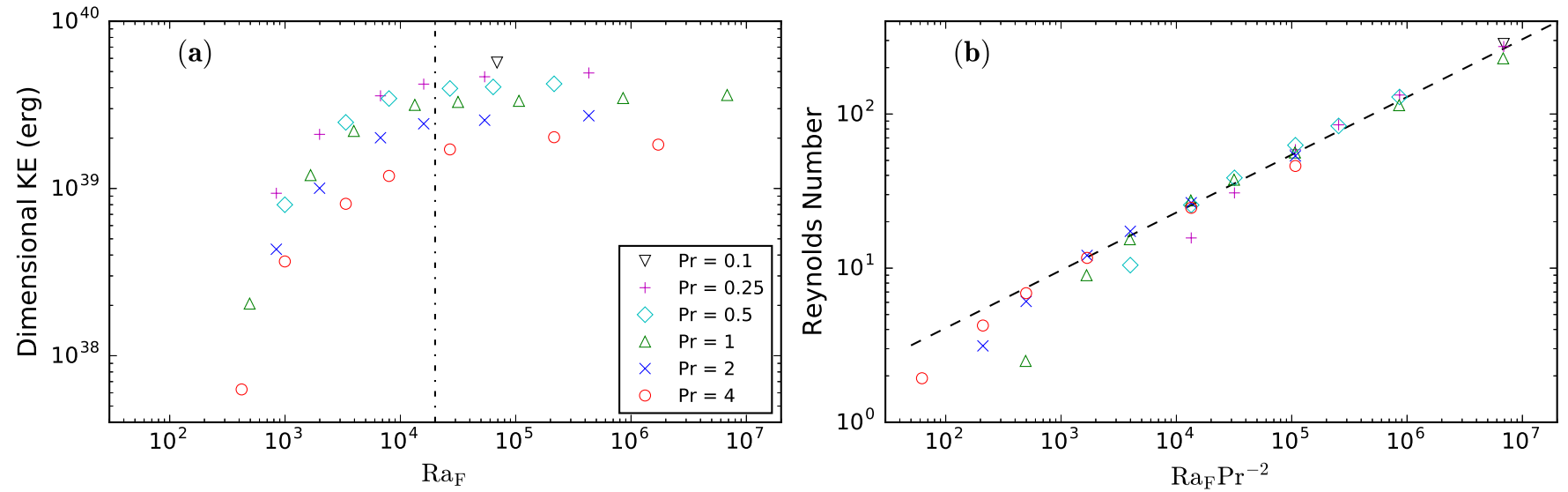

FIG. 1.- (a) Dimensional kinetic energy vs. flux Rayleigh number Ra $F$ for all cases. Colored symbols indicate different Prandtl numbers. Low Prandtl number runs have higher kinetic energies for a given $\mathrm{Ra}_{F}$. Beyond the high-Ra $\mathrm{F}_{F}$ cutoff, denoted by the vertical dot-dashed line, the kinetic energy tends toward an asymptotic value. (b) Reynolds number vs. $\operatorname{Ra}_{F} \operatorname{Pr}^{-2}$. The high-Ra $F$ region reaches an asymptotic regime with a power law scaling exponent that is very close to $1 / 3$. The dashed line is $\left(\operatorname{Ra}_{F} \operatorname{Pr}^{-2}\right)^{0.373}$. Colored symbols are the same as in panel (a).

Figure 1(a) shows the dimensional kinetic energy vs. $\mathrm{Ra}_{F}$. Different symbols indicate different values of Pr. As the Prandtl number is lowered, the kinetic energy increases for any given $\mathrm{Ra}_{F}$. The kinetic energy for those runs with $\operatorname{Ra}_{F} \gtrsim 2 \times 10^{4}$ appears to have reached a steady value that is independent of $\mathrm{Ra}_{F}$. The level at which the kinetic energy saturates is dependent on the Prandtl number. The saturation of the kinetic energy as the Rayleigh number is changed was also found in Featherstone \& Hindman (2016b), although their study was restricted to a Prandtl number of unity.

As $\operatorname{Pr}$ is increased, $\nu$ becomes larger leading to enhanced viscous dissipation and a smaller Reynolds number. The Reynolds number measures the relative ratio of inertial forces to viscous forces and is given by

$$
\operatorname{Re}=\frac{\sqrt{\widetilde{|\boldsymbol{u}|^{2}}} H}{\nu}
$$

where $H$ and the tilde retain the same meaning as before, representing the shell depth and a volume average, respectively. Larger Prandtl numbers will produce smaller Reynolds numbers for a given $\mathrm{Ra}_{F}$.

Figure 1(a) indicates that below some $\mathrm{Ra}_{F}$ cutoff, diffusion plays a leading-order role in the force balance. Beyond $\mathrm{Ra}_{F} \sim 2 \times 10^{4}$, which we denote the "high-Ra $\mathrm{R}_{F}$ " region ${ }^{1}$ this is no longer the case; diffusion no longer plays a leading-order role in the global force balance and the kinetic energy remains constant as $\mathrm{Ra}_{F}$ is increased. We note that the cutoff for the high- $\mathrm{Ra}_{F}$ region is based on the $\operatorname{Pr}=1$ results of Featherstone \& Hindman (2016b). Importantly, the cutoff is a decreasing function of the Prandtl number; lower Prandtl numbers will have a lower high-Ra $F$ cutoff. To simplify the analysis, only a single cutoff is used.

The kinetic energy can also be discussed from a nondimensional point of view. To do this, we choose a nondi-

1 This is the same cutoff used in Featherstone \& Hindman (2016b). Their reported $\mathrm{Ra}_{F}$ are too high by a factor of $\pi$; we correct for that here. mensional measure of the kinetic energy $\widehat{\mathrm{KE}}$ as

$$
\widehat{\mathrm{KE}} \equiv \frac{H^{2}}{\nu^{2} M} \mathrm{KE} .
$$

The nondimensionalization has been carried out using the mass $M$ contained within the spherical shell, the shell depth, and the viscous diffusion timescale. Under such a nondimensionalization, the kinetic energy can be related to the Reynolds number as

$$
\widehat{\mathrm{KE}} \sim \mathrm{Re}^{2} .
$$

Therefore we do not plot the nondimensional kinetic energy separately, but do list it for each run in Table 1.

If $\mathrm{Ra}_{F}$ is large enough to be in the high- $\mathrm{Ra}_{F}$ regime, then the kinetic energy is largely insensitive to the level of diffusion. This implies that the velocity is not strongly dependent on the level of diffusion and that the Reynolds number should scale with the viscosity as $\operatorname{Re} \sim \nu^{-1}$. Given our definition of $\mathrm{Ra}_{F}$ and $\mathrm{Pr}$, namely that $\mathrm{Ra}_{F} \sim$ $\nu^{-1} \kappa^{-2}$ and $\operatorname{Pr} \sim \nu \kappa^{-1}$, the Reynolds number scaling becomes $\operatorname{Re} \sim \operatorname{Ra}_{F}^{1 / 3} \operatorname{Pr}^{-2 / 3}$. In Figure $1(\mathrm{~b})$, we plot the Reynolds number vs. $\mathrm{Ra}_{F} \mathrm{Pr}^{-2}$, where the different symbols are the same as in panel (a). Each data point is time averaged. A least-squares fit to the data in the high- $\mathrm{Ra}_{F}$ region yields a scaling law of

$$
\operatorname{Re} \propto\left(\operatorname{Ra}_{F} \operatorname{Pr}^{-2}\right)^{0.373 \pm 0.008} .
$$

These results indicate that there are two distinct parameter regions; one in which diffusion is an important factor in the global force balance (the low- $\mathrm{Ra}_{F}$ region), and one where diffusion no longer plays an appreciable role in the interior, bulk global force balance (the high$\mathrm{Ra}_{F}$ region).

\subsection{Spectral Distribution}

We find the kinetic energy may reach a $\mathrm{Ra}_{F^{-}}$ independent regime, but the flow's morphology is still affected by the level of diffusion. This can be seen in the relative spectral distribution of velocity between the 

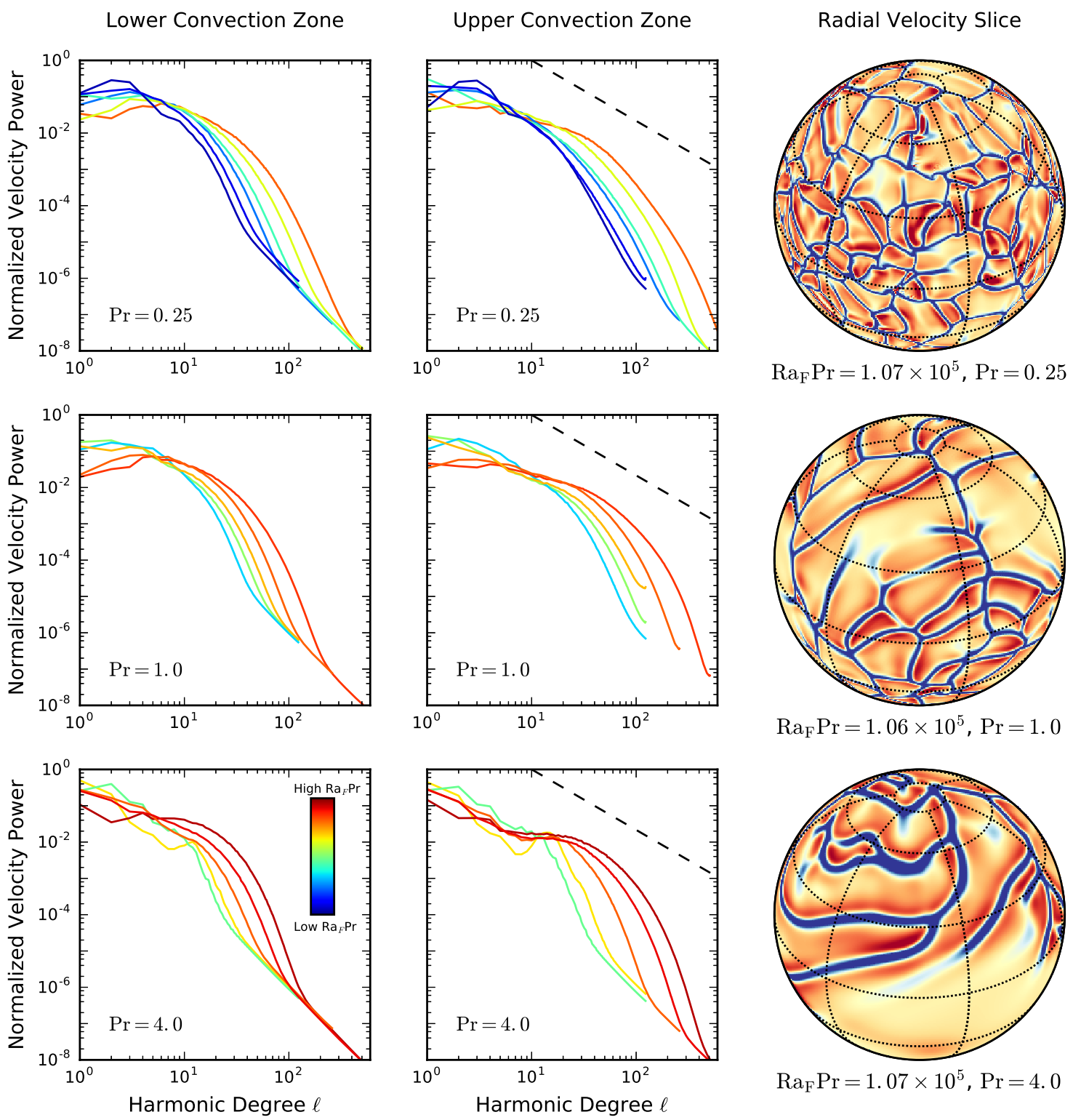

$\operatorname{Ra}_{\mathrm{F}} \operatorname{Pr}=1.07 \times 10^{5}, \operatorname{Pr}=4.0$

FIG. 2.- Time-averaged velocity power spectra and shell slices for cases with $\operatorname{Pr}=0.25, \operatorname{Pr}=1.0$, and $\operatorname{Pr}=4.0$. The power spectra have been normalized such that each curve has unit integrated power. Each row represents a different value of Pr. The first two columns correspond to power spectra at a single depth within the convective shell. The dashed black line in the center column has a slope of $-5 / 3$ for reference. Within each panel, spectra for all cases at that depth and Pr are displayed. Each curve is colored by $\mathrm{Ra}_{F} \operatorname{Pr}$ with low Ra ${ }_{F} \operatorname{Pr}$ in blue tones and high $\mathrm{Ra}_{F}$ cases are in red tones. As $\mathrm{Ra}_{F}$ is increased, the power at low $\ell$-values increases initially. At high RaF, it decreases as high-wavenumber power is generated at the expense of low-wavenumber power. This trend occurs at all Prandtl numbers studied. The last column shows shell slices of the radial velocity taken near the outer boundary at $r / r_{o} \approx 0.985$. The larger Pr run shows wider down flow lanes compared to the small Pr run.

high- and low-RaF systems. Figure 2 shows the velocity power spectra for all runs with Prandtl numbers of $\operatorname{Pr}=0.25, \operatorname{Pr}=1.0$, and $\operatorname{Pr}=4.0$. Each spectrum has been normalized such that it has unit integrated power. The rows correspond to the different Prandtl numbers. The first column shows the spectra taken at the lower convection zone or $r / r_{o} \approx 0.775$. The second column shows the spectra near the upper boundary, or $r / r_{o} \approx 0.985$, in the thermal boundary layer. In each panel, all spectra with the given Prandtl number are plotted. Each spectrum is colored by $\mathrm{Ra}_{F} \mathrm{Pr}$, with high values taking on red tones and low values displaying blue tones. Each spectrum is a time average over several tens of overturnings.

Across all cases, as $\mathrm{Ra}_{F}$ increases from low values, the point-wise velocity power increases at nearly all spherical harmonic degrees. The $\ell$ value associated with the peak for each spectra increases with increasing $\mathrm{Ra}_{F}$. This indicates smaller scale structures become more apparent with increasing $\mathrm{Ra}_{F}$. At sufficiently high-Rayleigh-number, power in the high- $\ell$ portion continues to increase, but the low- $\ell$ portion starts to decrease. This suggests a break down of large-scale coherent structures. The highest Rayleigh number runs have lower power in the large 

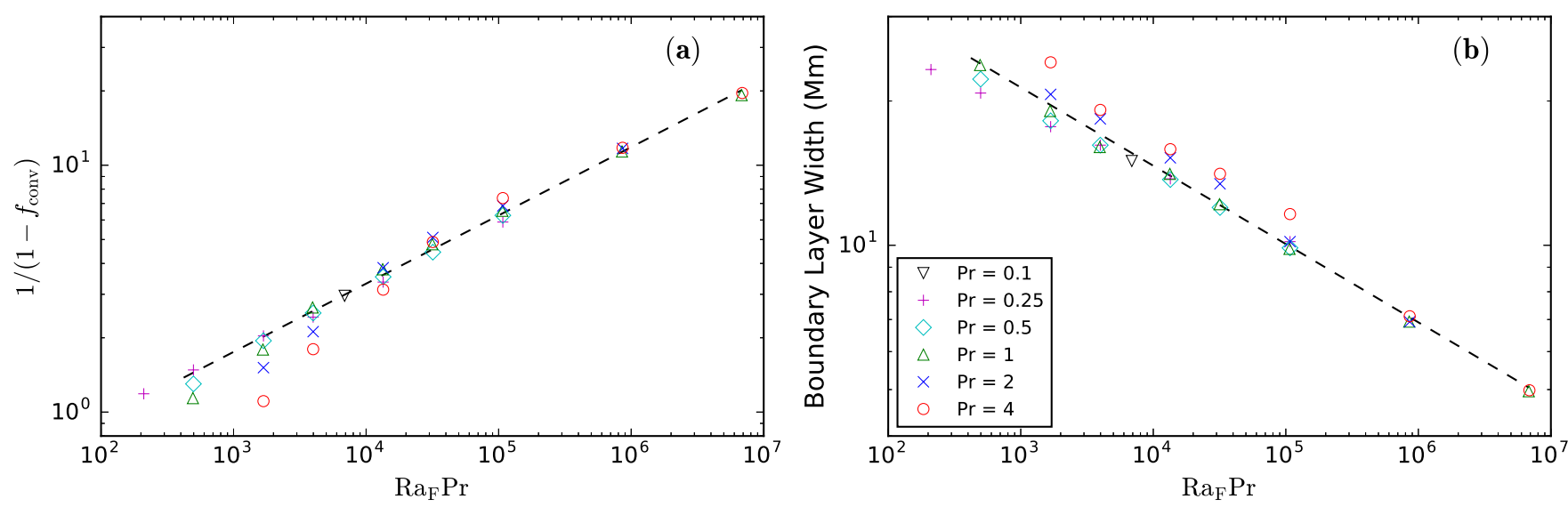

FIG. 3.- Energy transport and thermal boundary layer scaling with $\operatorname{Ra}_{F}$ Pr. (a) Fractional convective flux $1 /\left(1-f_{\text {conv }}\right)$ vs. Ra $\mathrm{Pr}_{F}$. The dashed line is $\left(\mathrm{Ra}_{F} \mathrm{Pr}\right)^{0.275}$, very close to a $2 / 7$ scaling. (b) Thermal boundary layer width plotted vs. RaF $\mathrm{Pr}$. The dashed line is $\left(\operatorname{Ra}_{F} \operatorname{Pr}\right)^{-0.164}$, very close to $-1 / 6$.

scales compared to the low-Ra $F$ counterparts. The "lowwavenumber" region occurs in the approximate range of $\ell \lesssim 10$ for most simulations in this study. This trend occurs for all values of $\operatorname{Pr}$ that were studied. If viscosity played a significant role in the asymptotic regime, one might expect there to be some variation in the spectra when the Prandtl number is varied. We do not observe large variations between the spectra for the Prandtl numbers within our range indicating that viscosity only plays a minor role in the asymptotic regime.

The third column in Figure 2 plots shell slices of the radial velocity taken near the upper boundary, the same location in radius as the second column. Each slice is a single snapshot in time. The color scale is the same for all three shell slices with red tones indicating positive, outward flows and blue tones indicating negative, inward flows. The lower Prandtl number run (the top panel) displays larger velocities and more small-scale structures compared to the larger Prandtl cases. The Reynolds numbers of all three slices cover a large range. The top row $\operatorname{Pr}=0.25$ run has $\operatorname{Re}=261.2$, the middle slice with $\operatorname{Pr}=1.0$ has $\operatorname{Re}=56.3$, and the bottom slice with $\operatorname{Pr}=4.0$ has $\operatorname{Re}=11.7$. The large range in Reynolds numbers indicates that the inertial subrange for each simulation is different.

At sufficiently high Rayleigh number, the integrated $\mathrm{KE}$ becomes independent of the level of diffusion (both thermal and momentum diffusion). As $\nu$ is decreased and $\mathrm{Ra}_{F}$ is increased, the flow becomes more turbulent with smaller scale structures. To leading-order, once the high$\mathrm{Ra}_{F}$ regime is reached, the total integrated dimensional $\mathrm{KE}$ is constant as the diffusion is further reduced. This fact is largely independent of Pr for the Prandtl numbers that were within our range, however, a weak Pr dependence remains because the inertial subrange is extended as the viscosity is reduced individually. These trends are similar to those found in the simultions of Featherstone \& Hindman (2016b), which used a Prandtl number of unity.

\subsection{Energy Transport}

The energy transport across the layer can be characterized by four radial energy fluxes: the enthalpy flux $F_{e}$, the kinetic energy flux $F_{\mathrm{KE}}$, the conductive flux $F_{c}$, and the viscous flux $F_{\nu}$, which we define as

$$
\begin{gathered}
F_{e}=\bar{\rho} c_{p} u_{r} T, \\
F_{\mathrm{KE}}=\frac{1}{2} \bar{\rho} u_{r}|\boldsymbol{u}|^{2}, \\
F_{c}=\kappa \bar{\rho} \bar{T} \frac{\partial S}{\partial r}, \\
F_{\nu}=-(\boldsymbol{u} \cdot \mathcal{D}) \cdot \hat{\boldsymbol{r}},
\end{gathered}
$$

respectively. Note that the conductive flux $F_{c}$ is associated with the diffusion of entropy perturbations, and it should not be confused with radiative diffusion arising from the reference state temperature gradient; that effect is represented by $Q$ in our models. Averages are taken of these fluxes over several diffusion times, indicated using brackets. We consider the contribution of conduction by looking at the fractional convective flux $f_{\text {conv }}$, defined as

$$
f_{\text {conv }} \equiv \frac{\int\left\langle F_{e}+F_{\nu}+F_{\mathrm{KE}}\right\rangle \mathrm{d}^{3} x}{\int\left\langle F_{c}+F_{e}+F_{\nu}+F_{\mathrm{KE}}\right\rangle \mathrm{d}^{3} x}
$$

Upon rearranging this quantity, we can write

$$
\begin{aligned}
\frac{1}{1-f_{\text {conv }}} & =\frac{\int\left\langle F_{c}+F_{e}+F_{\nu}+F_{\mathrm{KE}}\right\rangle \mathrm{d}^{3} x}{\int\left\langle F_{c}\right\rangle \mathrm{d}^{3} x} \\
& =1+\frac{\int\left\langle F_{e}+F_{\nu}+F_{\mathrm{KE}}\right\rangle \mathrm{d}^{3} x}{\int\left\langle F_{c}\right\rangle \mathrm{d}^{3} x}
\end{aligned}
$$

This resembles the traditional Nusselt number, but differs in two important ways. Firstly, the traditional Nusselt number does not include the viscous flux or the kinetic energy flux, both of which we include. Secondly, the conductive flux that appears in our definition is the established conductive flux, not the conductive flux in the absence of convection. Values of $1 /\left(1-f_{\text {conv }}\right)$ that are of order unity translate to a lack of convective heat transport. Large values indicate convection plays a dominant role over thermal conduction in transporting energy through the shell. Figure 3 (a) plots $1 /\left(1-f_{\text {conv }}\right)$ as a function of $\mathrm{Ra}_{F} \mathrm{Pr}$. Viscosity is not expected to play a large role in the heat transport across the shell, which is 


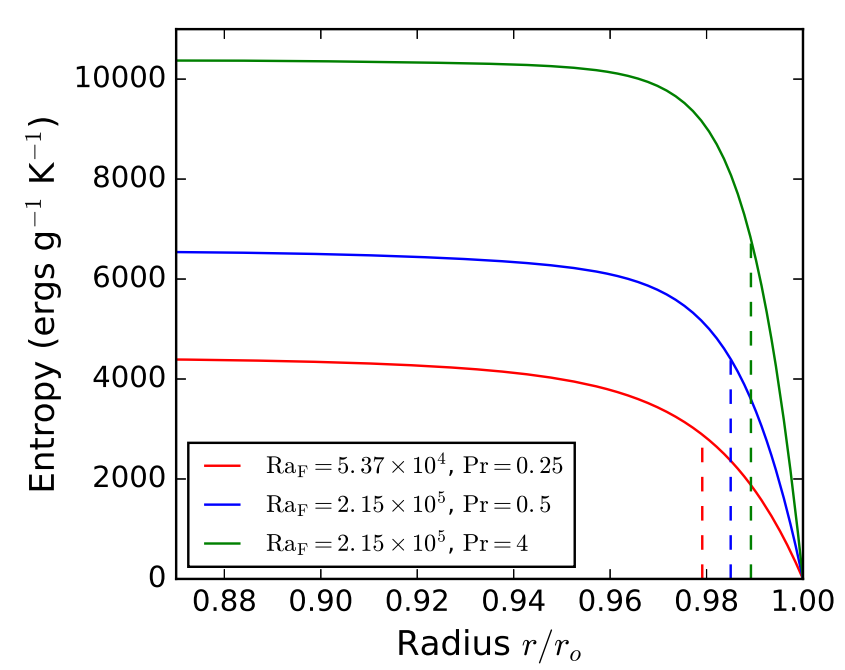

FIG. 4.- Time-averaged mean entropy profiles for three cases in the high-Ra $F$ regime. The vertical dashed lines indicate the location of the boundary layer as calculated using Equation 25

why we plot against $\mathrm{Ra}_{F} \mathrm{Pr}$ and not simply $\mathrm{Ra}_{F}$. The plot shows a clear trend that can be fit using least-squares to obtain

$$
\frac{1}{1-f_{\text {conv }}} \propto\left(\operatorname{Ra}_{F} \operatorname{Pr}\right)^{0.275 \pm 0.002}
$$

with a scaling exponent that is approximately $2 / 7(\sim$ 0.286 ). Other studies have found that the Nusselt number scales with the Rayleigh number to the $2 / 9$ power $(\sim 0.222)$ (e.g., Gastine et al. 2015), when a flux based Rayleigh number is used. Our results have a steeper exponent because we include the viscous flux and the kinetic energy flux, which act to increase $1 /\left(1-f_{\text {conv }}\right)$ at any given Rayleigh number.

\subsection{Boundary Layer Thickness}

We can further characterize the role of conduction by determining how $\mathrm{Ra}_{F}$ and Pr control the thermal boundary layer thickness. We define the thermal boundary layer thickness in terms of the time-averaged mean entropy, such that

$$
\delta_{\mathrm{BL}}=\int \frac{\sup \langle S(r)\rangle-\langle S(r)\rangle}{\sup \langle S(r)\rangle} \mathrm{d} r,
$$

where the brackets indicate a time-average as before and sup indicates the supremum. Figure 4 shows three examples of the time-averaged mean entropy profiles and the associated boundary layer location as calculated using Equation 25. We plot the variation of $\delta_{\mathrm{BL}}$ with $\mathrm{Ra}_{F} \mathrm{Pr}$ for different Prandtl numbers in Figure $3(\mathrm{~b})$. We plot against $\mathrm{Ra}_{F} \mathrm{Pr}$ because we do not expect the thermal boundary to depend on the viscosity and the quantity $\mathrm{Ra}_{F} \mathrm{Pr}$ is independent of the viscosity and scales as $\operatorname{Ra}_{F} \operatorname{Pr} \sim \kappa^{-3}$. A least-squares fit gives the scaling law

$$
\delta_{\mathrm{BL}} \propto\left(\mathrm{Ra}_{F} \mathrm{Pr}\right)^{-0.164 \pm 0.003} .
$$

The scaling exponent is very close to $-1 / 6(\sim-0.166)$, indicating that the boundary layer width scales purely as the thermal diffusivity, i.e., $\delta_{\mathrm{BL}} \propto \sqrt{\kappa}$. There is no strong dependence on viscosity within the range of $\mathrm{Pr}$ studied here.

We note that the highest Rayleigh number run had a boundary layer width of about $3 \%$ of the shell depth ( $\sim 5 \mathrm{Mm})$. Our thermal boundary layer lacks the radiative processes at work in a star like the Sun, but its physical extent is confined to a similarly small region of the convective domain.

Figure 1 showed that a free-fall regime that is independent of both viscosity and thermal diffusion could be obtained for different Prandtl numbers. We find that in order to have a bulk kinetic energy that is independent of both viscosity and thermal diffusion, the thermal boundary layer thickness must depend on both the Rayleigh number and the Prandtl number with the scaling law given in Equation 26 .

For a fixed Prandtl number, this scaling relation suggests that the boundary layer thickness will continually decrease as the Rayleigh number is increased. In global simulations that employ explicit diffusivities, the Prandtl number is therefore critical in maintaining a boundary layer that is confined to a small region of the convective domain without becoming vanishingly small as the Rayleigh number is increased.

Certainly, simulations with a conductive boundary layer cannot match every feature of the Sun's thermal boundary layer, for the simple reason that the Sun's boundary layer is regulated by radiative transfer instead of thermal conduction. In a global simulation without radiative transfer, one hopes that the microphysics of the cooling layer can be ignored and only the gross properties of the boundary layer (thickness and entropy contrast) are important. When the boundary layer is conductive, the entropy contrast and thickness are inherently linked. Thus, the best one could hope to do is achieve a physically realistic thickness; this requires that the product $H\left(\operatorname{Ra}_{F} \operatorname{Pr}\right)^{-1 / 6}$ take on the desired thickness, i.e., as the Rayleigh number is increased, the Prandtl number must be decreased. This realistic thickness will probably not, however, coincide with a convective power spectrum that possesses a realistic inertial range.

\section{PERSPECTIVES AND CONCLUSIONS}

The results presented here have interesting consequences for several aspects of stellar/solar convection zone dynamics. Many of these results will depend on rotation and magnetism, both of which were omitted in this study.

We find that simulations with a lower Pr number have faster flows and a broader range of scales compared to high $\mathrm{Pr}$ models with the same $\mathrm{Ra}_{F}$. The higher $\mathrm{Pr}$ models have more viscous dissipation, resulting in slower flows (equivalent to a lower Reynolds number). This is consistent with the results found in O'Mara et al. (2016).

The higher $\mathrm{Ra}_{F}$ simulations obtain a free-fall state where diffusion no longer plays an important role in the interior, bulk global force balance. In this state the kinetic energy becomes independent of both viscosity and thermal diffusion. Similar results were found in Featherstone \& Hindman (2016b), although their study was restricted to $\mathrm{Pr}=1$.

The boundary layer thickness scaling suggests that most simulations may be achieving the correct driving 
scale with a modest Rayleigh number of about $10^{6}$ and a Prandtl number of unity. The obtained scaling also suggests that in order to maintain a boundary layer whose physical extent is confined to a small region of the convective domain in a global simulation without radiative transfer, the Prandtl number should be decreased as the Rayleigh number is increased.

We did not find that the Prandtl number substantially alters the earlier observed behavior in the spectral distribution of the velocity for Pr unity simulations (e.g., Featherstone \& Hindman 2016b). As the $\mathrm{Ra}_{F}$ is increased, the convection develops smaller scale structures and a corresponding increase in the high-wavenumber power. The high-wavenumber power increases, but the low-wavenumber power decreases indicating the break down of coherent large-scale structures. The spectral range $\ell \leq 10$ appears to be the most sensitive region of the power spectrum. This occurs for all Prandtl numbers studied here.

Our results indicate that care needs to be taken when interpreting convection simulations and comparing the results to observations of real solar/stellar systems. Simulations that do not access a high enough $\mathrm{Ra}_{F}$, may overestimate the low-wavenumber power that is accessible to helioseismology. Stellar convection simulations must run with parameters that place it in the high-
Rayleigh-number regime in order to correctly capture the integrated kinetic energy and the large scale motions of the flow. We used rather modest levels of diffusion $\left(\kappa \leq 4 \times 10^{12} \mathrm{~cm}^{2} \mathrm{~s}^{-1}\right)$ to put our simulations in the high-Rayleigh-number regimes.

Most importantly, our simulations did not include rotation or magnetism. Featherstone \& Hindman (2016a) looked at the effects of rotation, but restricted their study to hydrodynamical, $\operatorname{Pr}=1$ simulations. It will be important to examine how our Prandtl number findings are modified by rotation and magnetism before we can fully trust comparisons of power spectra between observations and simulations.

This work was supported by NASA grants NNX09AB04G, NNX14AC05G, NNX11AJ36G, NNX17AM01G, and NNX14AG05G. Calkins was supported by the National Science Foundation award number EAR-1620649. Featherstone and the development of Rayleigh were further supported by the Computational Infrastructure for Geodynamics (CIG), which is supported by the National Science Foundation award numbers NSF-0949446 and NSF-1550901. Resources supporting this work were provided by the NASA High-End Computing (HEC) Program through the NASA Advanced Supercomputing (NAS) Division at Ames Research Center.

\section{REFERENCES}

Augustson, K. C., Brun, A. S., Miesch, M., \& Toomre, J. 2015, IAU General Assembly, 22, 2258283

Augustson, K. C., Brun, A. S., \& Toomre, J. 2016, ApJ, 829, 92

Bray, R. J., Loughhead, R. E., \& Durrant, C. J. 1984, The solar granulation (2nd edition)

Brown, B. P., Miesch, M. S., Browning, M. K., Brun, A. S., \& Toomre, J. 2011, ApJ, 731, 69

Brun, A. S., Strugarek, A., Varela, J., et al. 2017, ApJ, 836, 192

Calkins, M. A., Julien, K., \& Marti, P. 2015a, Proceedings of the Royal Society of London A: Mathematical, Physical and Engineering Sciences, 471, doi:10.1098/rspa.2014.0689

—. 2015b, Geophysical \& Astrophysical Fluid Dynamics, 109, 422

Chandrasekhar, S. 1961, Hydrodynamic and hydromagnetic stability

Christensen, U. R. 2011, Physics of the Earth and Planetary Interiors, 187, 157

Duarte, L. D. V., Wicht, J., Browning, M. K., \& Gastine, T. 2016 , Monthly Notices of the Royal Astronomical Society, 456, 1708

Featherstone, N. A., \& Hindman, B. W. 2016a, ApJ, 830, L15

-. 2016b, ApJ, 818, 32

Gastine, T., Wicht, J., \& Aubert, J. 2016, Journal of Fluid Mechanics, 808, 690

Gastine, T., Wicht, J., \& Aurnou, J. M. 2015, Journal of Fluid Mechanics, 778,721

Gilman, P. A. 1977, Geophysical and Astrophysical Fluid Dynamics, 8, 93

Gilman, P. A., \& Glatzmaier, G. A. 1981, ApJS, 45, 335

Goudard, L., \& Dormy, E. 2008, EPL (Europhysics Letters), 83 59001

Gough, D. O. 1969, Journal of Atmospheric Sciences, 26, 448

Greer, B. J., Hindman, B. W., Featherstone, N. A., \& Toomre, J. 2015, ApJ, 803, L17

Hanasoge, S. M., Duvall, T. L., \& Sreenivasan, K. R. 2012, Proceedings of the National Academy of Science, 109, 11928

Hart, A. B. 1954, MNRAS, 114, 17

Hathaway, D. H., Beck, J. G., Bogart, R. S., et al. 2000, Sol. Phys., 193, 299

Hathaway, D. H., Teil, T., Norton, A. A., \& Kitiashvili, I. 2015 ApJ, 811, 105
Jones, C. A. 2014, Icarus, 241, 148

Jones, C. A., Boronski, P., Brun, A. S., et al. 2011, Icarus, 216, 120

Käpylä, P. J., Mantere, M. J., \& Brandenburg, A. 2013

Geophysical and Astrophysical Fluid Dynamics, 107, 244

Leighton, R. B., Noyes, R. W., \& Simon, G. W. 1962, ApJ, 135, 474

Miesch, M. S., Brun, A. S., DeRosa, M. L., \& Toomre, J. 2008, ApJ, 673, 557

Miesch, M. S., Featherstone, N. A., Rempel, M., \& Trampedach R. 2012, ApJ, 757, 128

Nelson, N. J., Brown, B. P., Sacha Brun, A., Miesch, M. S., \& Toomre, J. 2014, Sol. Phys., 289, 441

O'Mara, B., Miesch, M. S., Featherstone, N. A., \& Augustson, K. C. 2016, Advances in Space Research, 58, 1475

Ossendrijver, M. 2003, A\&A Rev., 11, 287

Roberts, P. 2007, in Treatise on Geophysics, ed. G. Schubert (Amsterdam: Elsevier), $67-105$

Schrinner, M., Petitdemange, L., \& Dormy, E. 2012, ApJ, 752, 121

Schubert, G., Turcotte, D. L., \& Olson, P. 2001, Mantle Convection in the Earth and Planets (Cambridge University Press), doi:10.1017/CBO9780511612879

Soderlund, K. M., King, E. M., \& Aurnou, J. M. 2012, Earth and Planetary Science Letters, 333, 9

Spiegel, E. A. 1962, J. Geophys. Res., 67, 3063

Stein, R. F., Georgobiani, D., Schafenberger, W., Nordlund, Å., \& Benson, D. 2009, in American Institute of Physics Conference Series, Vol. 1094, 15th Cambridge Workshop on Cool Stars, Stellar Systems, and the Sun, ed. E. Stempels, 764-767

Thual, O. 1992, Journal of Fluid Mechanics, 240, 229

Ustyugov, S. D. 2010, Physica Scripta Volume T, 142, 014031

Wicht, J., \& Meduri, D. G. 2016, Physics of the Earth and Planetary Interiors, 259, 45 


\begin{tabular}{|c|c|c|c|c|c|c|c|c|c|c|c|}
\hline \multicolumn{6}{|c|}{ Input Parameters } & \multicolumn{6}{|c|}{ Output Parameters } \\
\hline $\begin{array}{l}\kappa \\
\left(10^{12}\right.\end{array}$ & $\begin{array}{c}\nu \\
\left.\mathrm{cm}^{2} \mathrm{~s}^{-1}\right)\end{array}$ & $\mathrm{Ra}_{F}$ & $\operatorname{Pr}$ & $n_{\max }$ & $\ell_{\max }$ & $\begin{array}{c}\mathrm{KE} \\
\left(10^{38} \text { erg }\right)\end{array}$ & $\widehat{\mathrm{KE}}$ & $f_{\text {conv }}$ & $\begin{array}{c}\delta_{\mathrm{BL}} \\
(\mathrm{Mm})\end{array}$ & $\operatorname{Re}$ & $\operatorname{Re}_{\text {peak }}$ \\
\hline 10 & 1 & $6.88 \times 10^{4}$ & 0.1 & 85 & 1023 & 34.43 & 19064.7 & 0.6620 & 15.57 & 248.3 & 342.1 \\
\hline 4 & 1 & $4.30 \times 10^{5}$ & 0.25 & 85 & 1023 & 42.59 & 23582.9 & 0.8301 & 10.36 & 261.2 & 376.9 \\
\hline 8 & 2 & $5.37 \times 10^{4}$ & 0.25 & 85 & 511 & 46.56 & 6446.4 & 0.7026 & 13.76 & 132.8 & 192.8 \\
\hline 12 & 3 & $1.59 \times 10^{4}$ & 0.25 & 85 & 511 & 42.08 & 2588.8 & 0.5876 & 16.18 & 85.1 & 123.4 \\
\hline 16 & 4 & $6.72 \times 10^{3}$ & 0.25 & 85 & 263 & 35.80 & 1238.4 & 0.5087 & 17.68 & 58.7 & 83.6 \\
\hline 24 & 6 & $1.99 \times 10^{3}$ & 0.25 & 85 & 127 & 21.07 & 324.8 & 0.3251 & 20.78 & 30.8 & 44.9 \\
\hline 32 & 8 & $8.40 \times 10^{2}$ & 0.25 & 85 & 127 & 9.36 & 81.6 & 0.1569 & 23.26 & 15.7 & 23.0 \\
\hline 4 & 2 & $2.15 \times 10^{5}$ & 0.5 & 85 & 511 & 42.24 & 5847.2 & 0.8403 & 9.87 & 129.0 & 186.0 \\
\hline 6 & 3 & $6.37 \times 10^{4}$ & 0.5 & 85 & 511 & 40.46 & 2489.6 & 0.7750 & 11.98 & 83.7 & 121.9 \\
\hline 8 & 4 & $2.68 \times 10^{4}$ & 0.5 & 85 & 263 & 39.62 & 1371.2 & 0.7167 & 13.71 & 62.7 & 91.9 \\
\hline 12 & 6 & $7.96 \times 10^{3}$ & 0.5 & 85 & 127 & 34.51 & 530.8 & 0.6031 & 16.18 & 38.5 & 56.5 \\
\hline 16 & 8 & $3.36 \times 10^{3}$ & 0.5 & 85 & 127 & 24.84 & 214.8 & 0.4860 & 18.18 & 25.6 & 38.4 \\
\hline 24 & 12 & $9.96 \times 10^{2}$ & 0.5 & 85 & 127 & 79.94 & 30.4 & 0.2314 & 22.21 & 10.4 & 16.5 \\
\hline 1 & 1 & $6.81 \times 10^{6}$ & 1 & 85 & 1023 & 36.22 & 20057.7 & 0.9480 & 4.96 & 229.8 & 320.4 \\
\hline 2 & 2 & $8.53 \times 10^{5}$ & 1 & 85 & 511 & 34.73 & 4807.6 & 0.9120 & 6.93 & 114.1 & 166.5 \\
\hline 4 & 4 & $1.06 \times 10^{5}$ & 1 & 85 & 263 & 33.42 & 1156.7 & 0.8470 & 9.83 & 56.3 & 85.6 \\
\hline 6 & 6 & $3.16 \times 10^{4}$ & 1 & 85 & 127 & 32.92 & 506.4 & 0.7910 & 12.18 & 37.5 & 58.2 \\
\hline 8 & 8 & $1.33 \times 10^{4}$ & 1 & 85 & 127 & 31.62 & 273.5 & 0.7360 & 14.09 & 27.5 & 42.4 \\
\hline 12 & 12 & $3.94 \times 10^{3}$ & 1 & 85 & 127 & 22.08 & 84.9 & 0.6230 & 16.03 & 15.4 & 24.0 \\
\hline 16 & 16 & $1.66 \times 10^{3}$ & 1 & 42 & 127 & 12.02 & 26.0 & 0.4420 & 19.06 & 9.0 & 15.6 \\
\hline 24 & 24 & $4.93 \times 10^{2}$ & 1 & 42 & 63 & 2.05 & 2.0 & 0.1220 & 23.77 & 2.5 & 4.5 \\
\hline 2 & 4 & $4.30 \times 10^{5}$ & 2 & 85 & 511 & 27.22 & 941.9 & 0.9145 & 6.92 & 53.5 & 78.0 \\
\hline 4 & 8 & $5.37 \times 10^{4}$ & 2 & 85 & 263 & 25.60 & 221.4 & 0.8539 & 10.18 & 26.6 & 40.0 \\
\hline 6 & 12 & $1.59 \times 10^{4}$ & 2 & 85 & 127 & 24.37 & 93.7 & 0.8038 & 13.43 & 17.3 & 26.2 \\
\hline 8 & 16 & $6.72 \times 10^{3}$ & 2 & 85 & 127 & 20.11 & 43.5 & 0.7398 & 15.22 & 12.1 & 18.7 \\
\hline 12 & 24 & $1.99 \times 10^{3}$ & 2 & 85 & 127 & 10.04 & 9.6 & 0.5277 & 18.35 & 6.1 & 9.9 \\
\hline 16 & 32 & $8.40 \times 10^{2}$ & 2 & 42 & 127 & 4.33 & 2.3 & 0.3393 & 20.65 & 3.1 & 5.2 \\
\hline 1 & 4 & $1.72 \times 10^{6}$ & 4 & 85 & 1023 & 17.10 & 591.8 & 0.9490 & 5.02 & 45.3 & 67.1 \\
\hline 2 & 8 & $2.15 \times 10^{5}$ & 4 & 85 & 511 & 20.26 & 175.2 & 0.9150 & 7.11 & 24.6 & 37.9 \\
\hline 4 & 16 & $2.68 \times 10^{4}$ & 4 & 85 & 263 & 17.12 & 37.0 & 0.8641 & 11.62 & 11.7 & 18.5 \\
\hline 6 & 24 & $7.96 \times 10^{3}$ & 4 & 85 & 127 & 11.88 & 11.4 & 0.7953 & 14.10 & 6.9 & 11.5 \\
\hline 8 & 32 & $3.36 \times 10^{3}$ & 4 & 85 & 127 & 8.10 & 4.3 & 0.6808 & 15.86 & 4.2 & 7.1 \\
\hline 12 & 48 & $9.96 \times 10^{2}$ & 4 & 85 & 127 & 3.66 & 0.8 & 0.4438 & 19.16 & 1.9 & 3.2 \\
\hline 16 & 64 & $4.29 \times 10^{2}$ & 4 & 42 & 127 & 0.63 & 0.1 & 0.0969 & 24.08 & 0.6 & 1.0 \\
\hline
\end{tabular}

TABLE 1

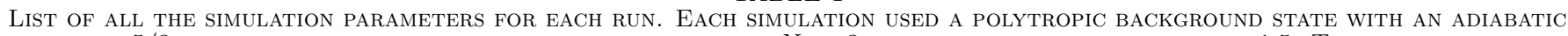
INDEX $\gamma=5 / 3$, THREE DENSITY SCALE HEIGHTS ACROSS THE DOMAIN $N_{\rho}=3$, AND A POLYTROPIC INDEX OF $n=1.5$. ThE INNER AND OUTER RAdi OF EACH Simulation Were $r_{i}=5 \times 10^{10}$ CM AND $r_{o}=6.586 \times 10^{10}$ CM. The Variable input parameters are the thermal

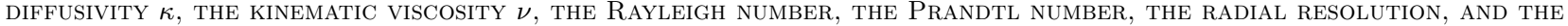
azimuthal Resolution. The output parameters are the dimensional Kinetic energy KE, nondimensional Kinetic energy KE, THE FRACTIONAL CONVECTIVE FLUX $f_{\text {conv }}$, THE DIMENSIONAL THERMAL BOUNDARY LAYER THICKNESS $\delta_{\text {BL, }}$, THE REYNOLDS NUMBER AND THE PEAK REYNOLDS NUMBER. 